\title{
PROPERTIES OF SOLUTIONS TO A SECOND ORDER NONLINEAR DIFFERENTIAL EQUATION
}

\author{
ALLAN J. KROOPNICK \\ Office of Retirement and Survivors Insurance \\ Social Security Administration \\ 3-D-22 Operations Building \\ 6401 Securlty Boulevard \\ Baltimore, Maryland 21235, U.S.A. \\ (Received August 22, 1989 and in revised form September 13, 1989)
}

ABSTRACT. In this note, we show when all solutions to the nonlinear differential equation $x^{\prime \prime}+c(t) f(x) g\left(x^{\prime}\right) x^{\prime}+a(t, x)=0$ are bounded. Furthermore, the solutions are either oscillatory or monotonic and asymptotically stable.

KEY WORDS AND PHRASES. Bounded, monotonic, oscillatory, asmyptotically Stable. 1980 AMS SUBJECT CLASSIFICATION CODES. Primary 34C11, 34C15.

1. INTRODUCTION.

In this note, we shall discuss under what conditions all solutions to the second order nonlinear differential equation,

$$
x^{\prime \prime}+c(t) f(x) g\left(x^{\prime}\right) x^{\prime}+a(t, x)=0
$$

are bounded and are either oscillatory or monotonic and approach 0 along with their derivatives as $t \rightarrow \infty$. By oscillatory, we shall mean a solution equaling 0 for arbitrarily large values of $t$. The above equation is a generalization of the wellknown Liênard equation (see, for example, Struble [1, p. 164-166] and Utz [2] for some classical results and Kroopnick [3] and Heidel [4,5] for some more recent though less general results to this type of equation). We now state and prove our main result.

2. MAIN RESULTS.

THEOREM 2.1. Given the differential equation (1.1). Suppose $C(.) \varepsilon C[0, \infty)$ and is positive on $[0, \infty)$ with $c_{1}>c(t)>0$ for some positive constant $c_{1}$ and $a(t, x)$ continuous on $[0, \infty) \times R$ with $x a(t, x)>a_{0}(t)|x|^{p}$ for $x \neq 0(p>1), a_{0}(t)$ positive and continuous on $[0, \infty)$ and $\int_{0}^{\infty} a_{0}(t) d t=+\infty$. Furthermore, suppose $x \frac{\partial}{\partial t} a(t, x)<0$, 
$\int_{x_{0}}^{ \pm} a(t, x) d x=+\infty$ uniformly in $t$ and left $f(\cdot), g(\cdot)$ be non-negative continuous functions on $R$. Then all solutions to (1.1) are bounded and are either oscillatory or monotonic and approach 0 along with their derivatives as $t \rightarrow \infty$.

PROOF. From standard existence theory, we know that a solution exists on the interval $[0, t)$ for some $t>0$. All we need to show is that the solution stays bounded and, therefore, it may be extended for all $t>0$. Now, multiply (1.1) by $x^{\prime}$ and integrate from 0 to $t$ by parts in order to obtain the following,

$$
\begin{aligned}
\frac{1}{2} x^{\prime 2}(t)+ & \int_{o}^{t} c(s) f(x(s)) g\left(x^{\prime}(s)\right) x^{\prime}(s)^{2} d s+\int_{x(o)}^{x(t)} a(t, u) d u \\
& -\int_{o}^{t} \int_{x(o)}^{x(s)} \frac{\partial}{\partial s} a(s, u(s)) d u d s=\frac{1}{2} x^{\prime 2}(0)
\end{aligned}
$$

(just differentiate (2.1) using Leibniz's rule to get back to (1.1)). Should $x$ and $x^{\prime}$ become unbounded then all terms on the LHS of (2.1) become positive and, furthermore, the LHS of (2.1) becomes unbounded which is impossible since the RHS of (2.1) is constant. A standard argument now completes the proof that $x(t)$ may be extended as a bounded solution for all $t>0$.

Next, suppose $x(t)$ does not oscillate, then $x(t)$ must eventually be of fixed sign for $t>\bar{t}>0$. Assume $x(t)>0$ for $t>\bar{t}$ (a similar argument works for $x(t)<0)$. Also, $x^{\prime}(t)$ must be of fixed sign. Otherwise, $x(t)$ will have an infinite number of consecutive relative maxima which is impossible if $x^{\prime}(t)=0$ infinitely often. In order to see this, suppose $x^{\prime}\left(t_{1}\right)$ and $x^{\prime}\left(t_{2}\right)$ are consecutive zeros of $x^{\prime}(t)$. Then $x\left(t_{1}\right)$ and $x\left(t_{2}\right)$ are consecutive relative maxima of $x(t)$ since $x^{\prime \prime}(t)<0$ at these points from (1.1). Also, the sign of $x^{\prime}$ does not change on $\left[t_{1}, t_{2}\right]$. Furthermore, we have $x^{\prime \prime}\left(t_{0}\right)=0$ where $t_{1}<t_{0}<t_{2}$. Should $x^{\prime}\left(t_{0}\right)>0$ then from (1.1) we have that $x^{\prime \prime}\left(t_{0}\right)<0$ which is impossible. If $x^{\prime}\left(t_{0}\right)<0$ then $x^{\prime}$ is negative on $\left(t_{1}, t_{2}\right)$ since $x^{\prime}$ does not change sign on $\left[t_{1}, t_{2}\right]$. However, if this is the case, $t$ hen $x(t)$ does not possess a relative maximum at $t=t_{2}$. This contradiction establishes that $x$ ' must eventually be of fixed sign.

Since $x(t)$ is positive, nonoscillatory and bounded, $x^{\prime}(t)$ must approach 0 as $t \rightarrow \infty$. Should $x(t)$ itself not approach 0 as $t \rightarrow \infty$, then we have $x^{\prime}(t) \rightarrow-\infty$ as $t \rightarrow \infty$. This follows from (1.1) when we integrate (1.1) from $\bar{t}$ to $t$, $1 . e$. ,

$$
x^{\prime}(t)=x^{\prime}(\bar{t})-\int_{\bar{t}}^{t} c(s) f(x(s)) g\left(x^{\prime}(s)\right) x^{\prime}(s) d s-\int_{\frac{t}{t}}^{t} a(s, x(s)) d s
$$

which diverges when $x(t)$ approaches a positive limit. Note that the middle term of the LHS of $(2.2)$ is bounded by $2 \mathrm{KMc}_{1}$ where $M$ is a bound for $f(x(t)) g\left(x^{\prime}(t)\right)$ and $K$ is a bound for $x(t)$ for $t \varepsilon[0,+\infty)$ so it cannot cancel out the third term of the RHS of (2.2) which diverges as $t+\infty$. 
We have essentially shown that the bounded solutions to (1.1) behave very much like those of $x^{\prime \prime}+c x^{\prime}+a x=0$ where a and $c$ are positive constants, an observation similar to one made by Utz [2].

EXAMPLE 2.1. Consider the nonlinear differential equation

$$
x^{\prime \prime}+c x^{2 m+1}+a x^{2 n+1}=0 \text { ( } m \text { and } n \text { are positive integers). }
$$

We readily see that all solutions to the above are bounded, oscillate or approach 0 from our theorem.

\section{REFERENCES}

1. STRUble, R.A., Nonlinear Differential Equations, McGraw-H111, New York, 1962.

2. UTZ, W.R., A Note on Second Order Nonlinear Differential Equations, Proc. Amer. Math. Soc. 7 (1956), 1047-1048.

3. KROOPNICK, A.J., Note on Bounded $\mathrm{L}^{\mathrm{P}}$-Solutions of a Generalized Lienard Equation, Pacific J. Math. 14 (1981), 171-175.

4. HEIDEL, J.W., Global Asymptotic Stability of a Generalized Lienard Equation, SIAM J. Appl. Math. 19 (1970), 629-637.

5. HEIDEL, J.W., A Liapunov Function for a Generalized Lienard Equation, J. Math. Anal. App1. $39(1972), 192-197$. 


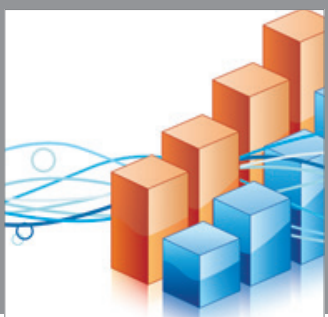

Advances in

Operations Research

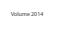

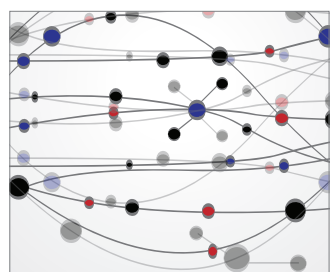

\section{The Scientific} World Journal
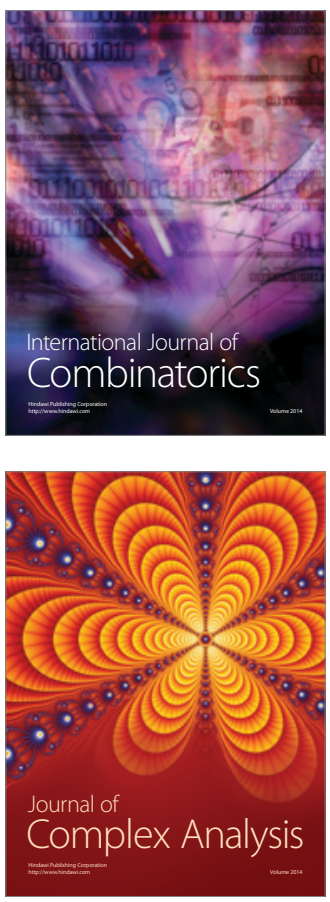

International Journal of

Mathematics and

Mathematical

Sciences
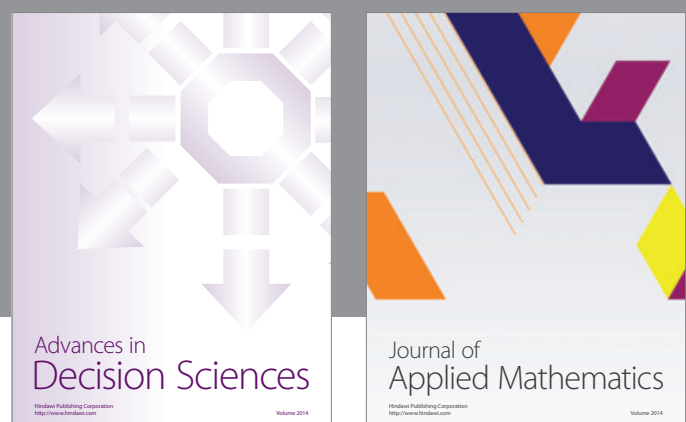

Journal of

Applied Mathematics
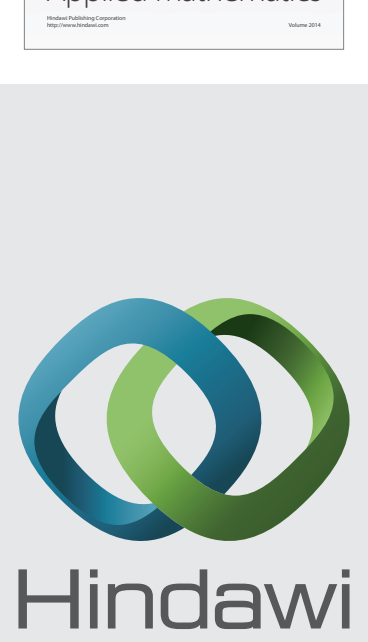

Submit your manuscripts at http://www.hindawi.com
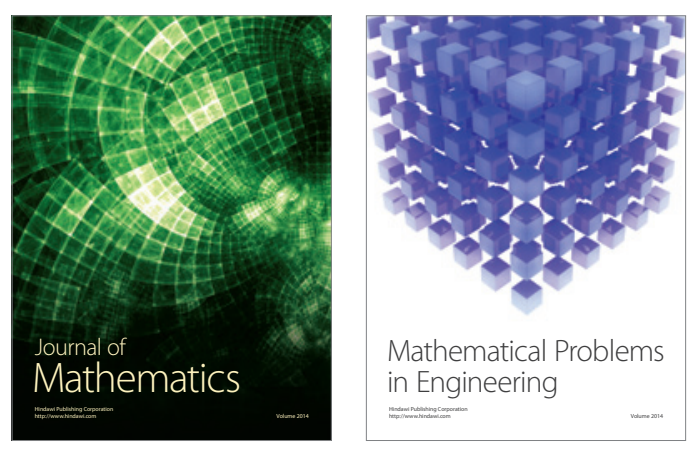

Mathematical Problems in Engineering
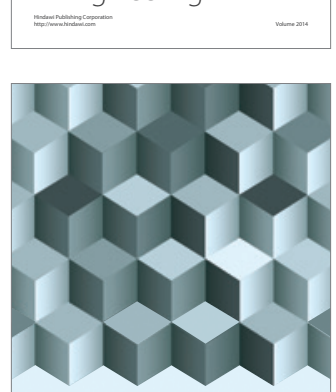

Journal of

Function Spaces
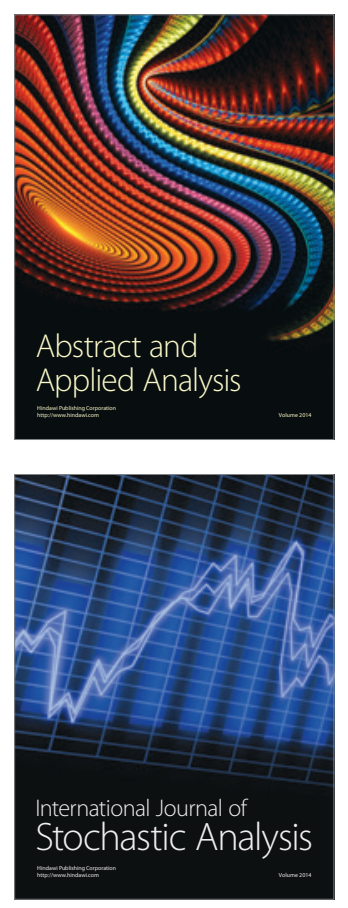

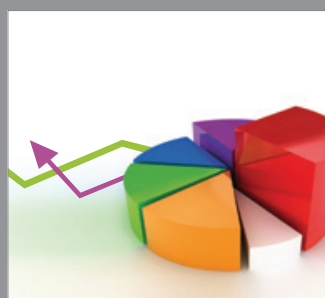

ournal of

Probability and Statistics

Promensencen
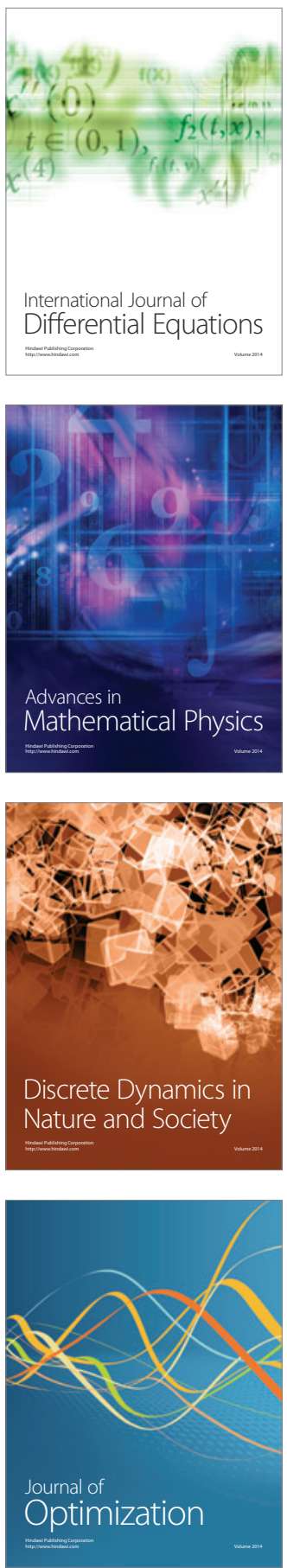